\title{
CONTRACÇÃO DIMENSIONAL SISTEMÁTICA: UMA PROPOSTA METODOLÓGICA PARA O CÁLCULO DE EQUAÇÕES E SISTEMAS DE $M$ EQUAÇÕES LINEARES COM $N$ INCÓGNITAS.
}

\author{
Francisco Lubota Bufeca Zau ${ }^{1}$ \\ https://orcid.org/0000-0003-4627-0375
}

\begin{abstract}
RESUMO
Os sistemas lineares homogéneos integram propriedades especiais que os diferenciam dos demais sistemas lineares e permitem simplificar a busca por soluções que, em certas condições, promovem soluções gerais de até sistemas heterogéneos e sistemas não lineares, daí a sua crucial importância na Matemática, ciências afins e na Engenharia. Desde os Nove Capítulos sobre a Arte Matemática da China Antiga a autores como SekiKowa, Leibniz, Cayley, Silvester, Bôcher, a resolução dos sistemas lineares passou a contar com métodos matriciais firmados em resultados teóricos como o algoritmo de eliminação de Gauss-Jordan, o teorema de Cramer, o teorema de Kronecker-Capelli. Introduziu-se, também, métodos iterativos clássicos como os de Jacobi-Richardson, Gauss-Seidel, factoração de Cholesck, o método SOR, métodos iterativos dos gradientes conjugados, assim como métodos gráficos. Contudo, este artigo apresenta uma alternativa metodológica inovadora denominada contracção dimensional sistemática, que não se funda em matrizes: visa, entre outras dinâmicas, reduzir, de forma sistemática, o número de incógnitas até que a respectiva resolução seja viável. Nesta visão, objectiva-se analisar a operacionalidade deste método de contracção dimensional sistemática no estudo de equações e sistemas lineares, a partir de técnicas homogéneas. Para o efeito, este artigo serve-se de uma pesquisa teórico-metodológica, de tipologia explicativa, com procedimentos técnicos bibliográficos e que utiliza o método indutivo - dedutivo. Assim, foi construído o método de contracção dimensional sistemática e aplicado para a obtenção de soluções originais e exactas de sistemas homogéneos de equações lineares, porque soluções desta natureza são condição necessária para a construção do produto vectorial homogéneo e, em geral, da Teoria Homogénea dos Espaços Vectoriais.
\end{abstract}

Palavras-chave: sistemas homogéneos; equações lineares; contracção dimensional sistemática.

Contracción dimensional sistemática: Una propuesta metodológica para el cálculo de ecuaciones y sistemas de $\boldsymbol{M}$ ecuaciones lineales con $\boldsymbol{N}$ incógnitas.

\section{RESUMEN}

Los sistemas lineales homogéneos integran propiedades especiales que los diferencian de los demás sistemas lineales y permiten simplificar la búsqueda por soluciones que, en ciertas condiciones, promueven soluciones generales de hasta sistemas heterogéneos y sistemas no lineales, de ahí su crucial importancia en la Matemática, ciencias afines y en la Ingeniería. Desde los Nueve Capítulos sobre el Arte Matemática de la Antigua China a los autores como SekiKowa, Leibniz, Cayley, Silvester, Bôcher, la resolución de los sistemas lineales pasó a contar con métodos matriciales firmados en resultados teóricos como el algoritmo de eliminación de Gauss-Jordan, el teorema de Cramer, el teorema de Kronecker-Capelli. Se introdujo también métodos iterativos clásicos como los de Jacobi-Richardson, Gauss-Seidel, factorización de Cholesck, el método SOR, métodos iterativos de los gradientes conjugados, así como métodos gráficos. En este artículo se presenta una alternativa metodológica innovadora denominada contracción dimensional sistemática, que no se fundamenta en matrices: busca, entre otras dinámicas, reducir, de forma sistemática, el número de incógnitas hasta que la respectiva resolución sea viable. En esta visión, se objetiva analizar la operacionalidad de este método de contracción dimensional sistemática en el estudio de ecuaciones y sistemas lineales, a partir de técnicas homogéneas. Para el efecto, este artículo se apoya en una pesquisa teórico-metodológica, de tipología explicativa, con procedimientos técnicos bibliográficos y que utiliza el método inductivo - deductivo. Así, fue construido la propuesta metodológica del método de contracción dimensional sistemática y aplicado para la obtención de soluciones originales y exactas de sistemas homogéneos de ecuaciones lineales, porque soluciones de esta naturaleza son condición necesaria para la construcción del producto vectorial homogéneo y, en general, de la Teoría Homogénea de los Espacios Vectoriales.

${ }^{1}$ Doutorando em Gestão de Empresas pelo Programa de Pós-graduação da Universidade San Lourenzo de Paraguay (UNISAL). Director Geral Adjunto para os Assuntos Científicos do Instituto Superior Politécnico do Cazenga (ISPOCA). Email: zaufrancisco@gmail.com 
Palabras clave: sistemas homogéneos; ecuaciones lineales; contracción dimensional sistemática.

Systematic dimensional contracting: A methodological proposal for the calculation of equations and systems of $\boldsymbol{M}$ linear equations with $\boldsymbol{N}$ unknowns.

\section{ABSTRACT}

Homogeneous linear systems integrate special properties that differentiate them from other linear systems and allow to simplify the search for solutions that, under certain conditions, promote general solutions of even heterogeneous systems and non-linear systems, hence their crucial importance in Mathematics, related sciences and in Engineering. From the Nine Chapters on the Mathematical Art of Ancient China to authors such as Seki Kowa, Leibniz, Cayley, Silvester, Bôcher, the resolutions of linear systems started to rely on matrix methods based on theoretical results such as the Gauss-Jordan elimination algorithm, Cramer's theorem, the Kronecker-Capelli theorem. Classic iterative methods such as Jacobi-Richardson, Gauss-Seidel, Cholesck factorization, the SOR method, conjugate gradient iterative methods, as well as graphical methods were also introduced. However, this article presents an innovative methodological alternative called systematic dimensional contraction, which is not based on matrices: it aims, among other dynamics, to systematically reduce the number of unknowns until the respective resolution is viable. In this view, the objective is to analyze the operability of this method of systematic dimensional contraction in the study of equations and linear systems, using homogeneous techniques. For this purpose, this article makes use of a theoreticalmethodological research, of an explanatory typology, with bibliographic technical procedures and that uses the inductive-deductive method. Thus, the methodologic proposal for a systematic dimensional contraction method was constructed and applied to obtain original and exact solutions of homogeneous systems of linear equations, because solutions of this nature are a necessary condition for the construction of the homogeneous vector product and, in general, of the homogeneous theory of Vector Spaces.

Keywords: homogeneous systems; linear equations; systematic dimensional contraction.

\section{Introdução}

As equações lineares e sistemas de equações lineares constituem objectos matemáticos omnipresentes em problemas científicos e tecnológicos enfrentados pela Humanidade, e surgem naturalmente em várias ciências para as quais o conhecimento matemático é significativo, tais como Física, Química, Engenharia, Economia, Astronomia, Cosmologia, Ciências de Computação, Biologia, dentre outras (Santos, 2010). Assim, achar um conjunto-solução de equações lineares ou sistemas de equações lineares constitui um imperativo crítico para a resolução de problemas científicos, técnicos, profissionais e metodológicos.

Existem métodos destinados à resolução de sistemas de equações lineares nos campos da Álgebra Linear e da Geometria Analítica. Estes métodos dividem-se em dois grandes grupos: métodos directos e os métodos iterativos. Os métodos directos são aqueles que fornecem soluções analíticas exactas relativas a sistemas lineares, caso tais soluções existam. Os métodos iterativos geram uma sequência de vectores a partir de uma aproximação inicial, convergindo para a solução exacta do sistema linear, caso exista (Gonçalves, 2018). Dentre os métodos directos contam-se o método de substituição progressiva e retroactiva, o método de eliminação de Gauss, o método de decomposição LU, o método de Gauss-Jordan, o método de Cholesky. Dentre os métodos iterativos constam o método de Jacobi-Richardson, o método de Gauss-Seidel, o método dos gradientes conjugados.

$\mathrm{Na}$ resolução de sistemas lineares homogéneos com $m$ equações e $n$ incógnitas, para casos em que $n>m$, pelo método geral de escalonamento, surge, com frequência, uma anomalia de cálculo aparentemente imperceptível: a presença de variáveis livres, as quais podem assumir quaisquer valores dentro de dado conjunto numérico. A anomalia de cálculo em questão está relacionada com o facto de a consideração de variáveis livres mitigar a verdadeira estrutura algébrica das soluções do sistema linear homogéneo, perdendo-se assim informações importantes sobre o verdadeiro layout das soluções exactas destes sistemas lineares homogéneos, não obstante numericamente oferecer soluções viáveis para o sistema linear proposto.

Para contornar o uso de variáveis livres e permitir a obtenção de soluções gerais e exactas, algébrica e estruturalmente adequadas no cálculo de equações e sistemas lineares com $m$ equações e $n$ 
incógnitas, o autor deste artigo propõe a utilização do método de contracção dimensional sistemática de equações e sistemas lineares. Trata-se de um método que resulta de uma aplicação directa de um resultado da Álgebra Linear apresentado na proposição 3 que se segue mais adiante. Destarte, constitui objectivo geral deste artigo apresentar uma proposta metodológica denominada contracção dimensional sistemática, sua operacionalização e vantagens no cálculo de equações e sistemas homogéneos de equações lineares com $m$ equações e $n$ incógnitas. Deste objectivo genérico se determinaram os seguintes objectivos específicos:

- Relacionar equações lineares e sistemas homogéneos de equações lineares;

- Definir, em conformidade com a proposta metodológica aqui apresentada, a equação homogénea geral;

- Abordar sobre equações homogéneas lineares identitárias e vectoriais;

- Descrever as etapas e operacionalidade do método de contracção dimensional sistemática no cálculo de equações e sistemas de equações lineares.

Por estes objectivos específicos, este artigo integra as seguintes temáticas: introdução ao estudo da equação homogénea linear, equação linear e sistemas homogéneos, equação homogénea linear identitária, equação homogénea linear vectorial, resolução de equações homogéneas lineares pelo método de contracção dimensional sistemática, resolução de sistemas de equações homogéneas lineares pelo método de contracção dimensional sistemática, solução-padrão de equação homogénea e sistema homogéneo de equações lineares.

\section{Caracterização das Equações Lineares e dos Sistemas Homogéneos das Equações Lineares}

A caracterização das equações lineares e dos sistemas homogéneos das equações lineares apresenta uma temática algébrica vinculada ao conceito de equação homogénea linear, que difere, no campo conceptual, da ideia associada à equação linear homogénea, bastante difundida na Álgebra Linear, na qual uma equação linear da forma $A \boldsymbol{x}=\boldsymbol{b}$ é tal que $A$ é a matriz dos coeficientes, $\boldsymbol{x}$ é o vector de incógnitas e $\boldsymbol{b}$, o vector de termos independentes (Steinbruch e Winterle, 1995), conforme segue.

$$
\left\{\begin{array}{c}
a_{11} x_{1}+a_{12} x_{2}+a_{13} x_{3}+\cdots+a_{1 n} x_{n}=b_{1} \\
a_{21} x_{1}+a_{22} x_{2}+a_{23} x_{3}+\cdots+a_{2 n} x_{n}=b_{2} \\
\cdots \cdots \cdots \cdots \cdots \cdots \cdots \cdots \cdots \cdots \cdots \cdots \cdots \cdots \cdots \cdots \cdots \cdots \cdots \cdots \cdots \cdots \cdots \cdots \cdots \cdots \cdots \cdots \cdots \cdots \cdots \cdots \cdots \cdots \cdots \cdots \cdots \cdots \cdots \cdots \cdots \cdots \cdots \cdots \cdots \cdots \cdots \cdots \cdots \cdots \cdots \cdots \cdots \cdots \cdots \cdots \cdots \\
\cdots \cdots \cdots \cdots \cdots \cdots \cdots
\end{array}\right.
$$

A Álgebra Linear, que estuda matrizes, sistemas lineares e tópicos relacionados (Anton e Rorres, 2012), demonstra que toda a informação necessária para a resolução de um sistema de equações lineares está contida na respectiva matriz $A$ que, no caso do sistema linear acima, segue abaixo.

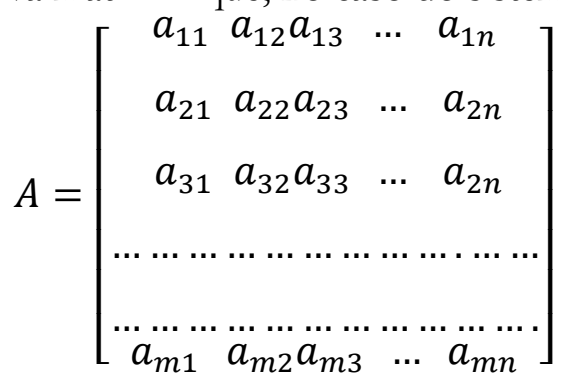

Uma matriz de coeficientes é uma matriz $m \times n$ cujas linhas são coeficientes $a_{i j}$ de equações lineares de um sistema linear ou transformação linear. A sua operacionalização requer domínio de 
técnicas da Álgebra Matricial e constituem objectos matemáticos muito importantes no contexto profissional, porquanto muitos problemas reais na engenharia, economia, ciências naturais e até na Matemática podem ser transformados em sistemas de equações lineares (Cabral e Goldfeld, 2012).

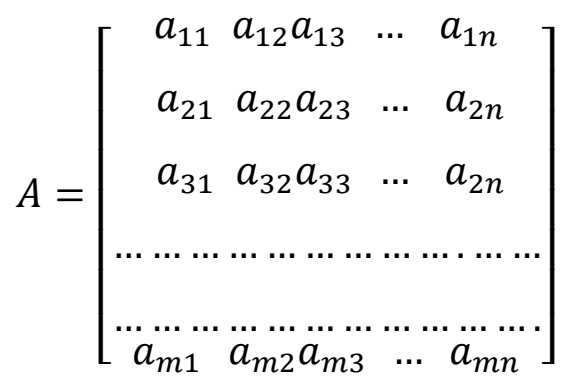

Assim, a solução de um sistema linear, como o apresentado acima, pode ser obtida efectuando operações apropriadas nessa matriz, denominadas operações elementares, dentre outras, compiladas em métodos directos, como os já referenciados. Em particular, considerando o teorema de Kronecker-Capelli, um sistema linear $A \boldsymbol{x}=\boldsymbol{b}$, com característica $c(A)$ e matriz aumentada $A \mid \boldsymbol{b}$, é compatível se e somente se $c(A)=c(A \mid \boldsymbol{b})$ e, em caso de ser compatível, será determinado para $c(A)=n$ e indeterminado para $c(A)<n$.

A Álgebra Homométrica estuda estruturas algébricas onde estão definidas duas aplicações multilineares como produtos da álgebra, uma transformando espaços vectoriais em outro espaço vectorial denominado espaço homogéneo, e outra aplicando estes espaços vectoriais no corpo de escalares sobre o qual tais espaços estão definidos. Esta álgebra aproveita as propriedades especiais e exclusivas de equações e sistemas lineares homogéneos para obter soluções exactas mais simples destes sistemas. E, sistemas lineares não homogéneos são contemplados nestes procedimentos, devendo ser previamente convertidos em sistemas homogéneos. O conjunto de técnicas que servem esta finalidade constitui o método de contracção dimensional sistemática abordado neste artigo, assim denominado por colocar o foco na redução sistemática do número de incógnitas da equação ou sistema de equações lineares homogéneo dado, até atingir um número adequado a uma resolução mais viável, que não recorra ao uso de variáveis livres.

Considere-se o seguinte sistema de duas equações lineares com três incógnitas.

$$
\left\{\begin{array}{l}
a_{1} x_{1}+a_{2} x_{2}+a_{3} x_{3}=c_{1} \\
b_{1} x_{1}+b_{2} x_{2}+b_{3} x_{3}=c_{2}
\end{array}\right.
$$

Neste sistema, as incógnitas são $x_{1}, x_{2}, x_{3}$; os coeficientes são $a_{1}, a_{2}, a_{3}$ e $b_{1}, b_{2}, b_{3}$; os termos independentes são $c_{1}, c_{2}$. Impondo ao sistema (1) uma interpretação vectorial, segue que $c_{1}=$ $a_{1} x_{01}+a_{2} x_{02}+a_{3} x_{03}$ e $c_{2}=b_{1} x_{01}+b_{2} x_{02}+b_{3} x_{03}$. Substituindo estas expressões no sistema (1), tem-se o seguinte sistema homogéneo:

$$
\text { II }\left\{\begin{array}{l}
a_{1}\left(x_{1}-x_{01}\right)+a_{2}\left(x_{2}-x_{02}\right)+a_{3}\left(x_{3}-x_{03}\right)=0 \\
b_{1}\left(x_{1}-x_{01}\right)+b_{2}\left(x_{2}-x_{02}\right)+b_{3}\left(x_{3}-x_{03}\right)=0
\end{array}\right.
$$

Este sistema (2) pode obter-se a partir de situações concretas, como por exemplo, na Gestão Empresarial (Silva et al, 2020). Considere-se, para o efeito, a seguinte relação financeira:

\section{Vendas $=$ Custos Fixos + Custo Variável + Lucro Líquido.}

Represente-se as parcelas desta igualdade por variáveis do ponto de equilíbrio para as quais $c_{1}$ e $c_{2}$ são os valores de vendas do primeiro exercício económico e do segundo exercício económico, respectivamente. Obviamente, denotando por $x_{1}$ o valor dos Custos Fixos no ponto de equilíbrio, 
pode-se denotar por $a_{1} x_{1}$ e $b_{1} x_{1}$ os Custos Fixos no primeiro exercício económico e no segundo exercício económico da empresa considerada, respectivamente. Repetindo este processo para as demais parcelas da equação acima, tem-se o seguinte sistema linear, no qual as constantes reais $a_{1}$, $a_{2}, a_{3}$ e $b_{1}, b_{2}, b_{3}$ exprimem uma comparação de variáveis económicas desta equação no instante do ponto de equilíbrio em relação àqueles exercícios económicos.

$$
\left\{\begin{array}{l}
a_{1} x_{1}+a_{2} x_{2}+a_{3} x_{3}=c_{1} \\
b_{1} x_{1}+b_{2} x_{2}+b_{3} x_{3}=c_{2}
\end{array}\right.
$$

Considerando uma interpretação vectorial para este sistema, chega-se ao sistema (2) acima, o qual pode ser resolvido para se encontrar os valores reais referenciadas anteriormente.

O sistema homogéneo (2) acima pode originar dois subsistemas homogéneos, chamados sistemas parciais, agrupando equações semelhantes, de tal modo que cada um destes subsistemas seja derivado de uma equação do sistema homogéneo original (2), ou seja:

$$
\mathrm{I}\left\{\begin{array} { l } 
{ a _ { 1 } x _ { 0 1 } + a _ { 2 } x _ { 0 2 } + a _ { 3 } x _ { 0 3 } = 0 } \\
{ a _ { 1 } x _ { 1 } + a _ { 2 } x _ { 2 } + a _ { 3 } x _ { 3 } = 0 }
\end{array} \quad \mathrm { e } \quad \mathrm { II } \left\{\begin{array}{l}
b_{1} x_{01}+b_{2} x_{02}+b_{3} x_{03}=0 \\
b_{1} x_{1}+b_{2} x_{2}+b_{3} x_{3}=0
\end{array}\right.\right.
$$

Destes sistemas homogéneos lineares (3) vê-se, com toda a clareza, que houve inversão de papéis em relação aos elementos do sistema (1), ou seja, as incógnitas passaram a ser, na equação I, $a_{1}$, $a_{2}, a_{3}$, e na equação II, $b_{1}, b_{2}, b_{3}$, elementos que originalmente eram considerados coeficientes no sistema (1); as incógnitas do sistema (1) passaram a ser, nestes sistemas (3), coeficientes.

A interpretação vectorial de um sistema de equações lineares permite identificar três vectores: um vector inicial, um vector pós-inicial, ambos dados, e um vector de coeficientes que deverá ser determinado (Santos, 2010). Geometricamente, o vector de coeficientes é ortogonal aos outros dois vectores, pelo que, a rigor, não permite a recuperação de um dos outros dois vectores, ou seja, este vector de coeficientes não pode ser dado a priori para recuperar um dos outros dois.

Pelo observado acima, Álgebra Homométrica considera que um sistema homogéneo, matricialmente dado na forma $A \boldsymbol{x}=\mathbf{0}$, é tal que $A$ representa a matriz das coordenadas de vectores $\boldsymbol{v}_{i} \in \mathbb{V}^{n}$, para $i, n \in \mathbb{N}$, e $\boldsymbol{x}$, o vector dos coeficientes de uma equação linear definida pelos vectores $\boldsymbol{v}_{i}$ deste espaço vectorial $\mathbb{V}^{n}$, de dimensão finita, sobre um corpo de escalares $\mathbb{K}$. Pelo exposto acima, $x_{1}, x_{2}, x_{3}, \ldots, x_{n} \in \mathbb{K}$ e $\boldsymbol{x} \in \mathbb{V}^{n}$, ou seja, tal como acontece no contexto da Álgebra Linear, $\boldsymbol{x}$ é um vector de $\mathbb{V}^{n}$ composto por escalares do corpo $\mathbb{K}$. Esta interpretação algébrica da Álgebra Homométrica será desenvolvida em trabalhos futuros, aquando da abordagem sobre o espaço vectorial homogéneo.

Ademais, o aspecto sistémico da equação matricial $A \boldsymbol{x}=\mathbf{0}$ não difere da sua forma algébrica conhecida, sendo que a primeira diferença formal é de perspectiva, ou seja, a forma como este sistema homogéneo é interpretado na visão da Álgebra Linear e na perspectiva da Álgebra Homométrica: nesta última, as incógnitas de $A \boldsymbol{x}=\mathbf{0}$ são componentes de um vector $\boldsymbol{x}$, cujos coeficientes formam a matriz $A$; na visão de todo investigador interessado ao estudo do referido fenómeno, as incógnitas são coeficientes de uma equação linear vectorial que relaciona os vectores $\boldsymbol{v}_{i} \in \mathbb{V}^{n}$ e que compõem o vector $\boldsymbol{x}$, sendo os componentes destes vectores $\boldsymbol{v}_{i} \in \mathbb{V}^{n}$ elementos da matriz $A$ deste sistema homogéneo.

Por conseguinte, para resolver o sistema homogéneo $A \boldsymbol{x}=\mathbf{0}$ é preciso garantir que os vectores $\boldsymbol{v}_{i}$ do espaço vectorial $\mathbb{V}^{n}$ existem e são conhecidos, cujas características combinadas produzem o vector dos coeficientes que pode ser compreendido como único vector linearmente independente que resulta da operação linear vectorial dos dois vectores multiplicativos $\boldsymbol{v}_{i} \in \mathbb{V}^{n}$. 


\section{Estudo da Aplicação das Equações Homogéneas Gerais}

O conceito de equação homogénea não é novo em Matemática e suas disciplinas associadas, sendo bastante familiar em campos como Álgebra Linear e Equações Diferenciais. No contexto de Álgebra Linear, as equações homogéneas constituem uma variante das equações lineares e, considerando um espaço vectorial $\mathbb{V}^{m}$ sobre um corpo $\mathbb{K}$, uma equação linear homogénea na Álgebra Linear pode ser expressa pela igualdade:

$$
\sum_{i=1}^{n}\left(b_{i} \boldsymbol{x}_{i}\right)=0, \quad \forall b_{i} \in \mathbb{K}, \boldsymbol{x}_{i} \in \mathbb{V}^{n}
$$

Os elementos $b_{i}$ são denominados constantes ou coeficientes da equação linear homogénea, enquanto os elementos $\boldsymbol{x}_{\boldsymbol{i}}$ são chamados de incógnitas da mesma equação, que podem ser vectores, tal como expresso nesta igualdade acima, ou podem ser quaisquer variáveis que representem parâmetros ou números não conhecidos. No caso de $\boldsymbol{x}_{i}$ serem vectores, a equação (1) é dita vectorial e, se $x_{i}$ são variáveis escalares, esta equação (1) é denominada escalar.

De modo menos compacto, a equação linear homogénea (1) pode ser escrita na forma extensiva, sem o uso do sinal de somatório, tal como segue abaixo.

$$
b_{1} x_{1}+b_{2} x_{2}+b_{3} x_{3}+\cdots+b_{n} x_{n}=0
$$

O conjunto $Y=\left\{y_{1} ; y_{2} ; y_{3} ; \ldots ; y_{n}\right\}$ é chamado solução da equação linear homogénia (1) se satisfaz a igualdade respectiva, ou seja, $b_{1} y_{1}+b_{2} y_{2}+b_{3} y_{3}+\cdots+b_{n} y_{n}=0$, de maneira que se tenha:

$$
x_{1}=y_{1}, x_{2}=y_{2}, x_{3}=y_{3}, \ldots, x_{n}=y_{n} .
$$

Um conjunto de equações lineares homogéneas pode formar um sistema de equações lineares homogéneas, passando o respectivo conjunto-solução a satisfazer, ao mesmo tempo, todas as equações que compõem o respectivo sistema de equações lineares homogéneas. Ressalta-se que, todo sistema linear homogéneo é classificado como possível determinado, ou seja, tem sempre solução que pode ser apenas a solução trivial ou infinitas soluções, conforme o teorema de Kronecker-Capelli (Castro, 2018). Neste caso, um sistema de equações lineares homogéneas tem a seguinte forma:

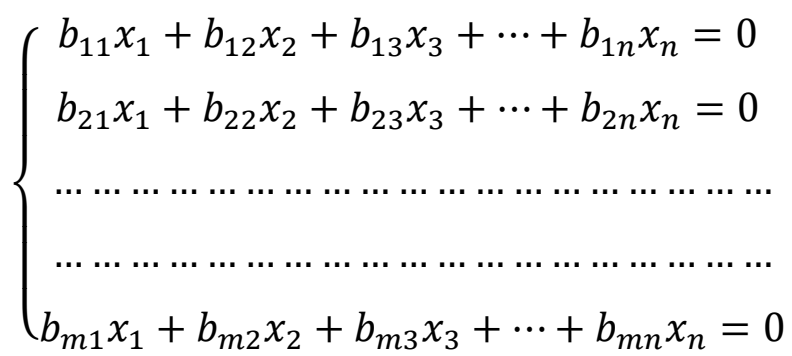

Este sistema homogéneo pode ser escrito na forma matricial, ou seja, $A \boldsymbol{x}=\mathbf{0}$, atendendo à forma matricial de um sistema linear $\boldsymbol{A} \boldsymbol{x}=\boldsymbol{C}$, onde, neste caso, tem-se conforme abaixo.

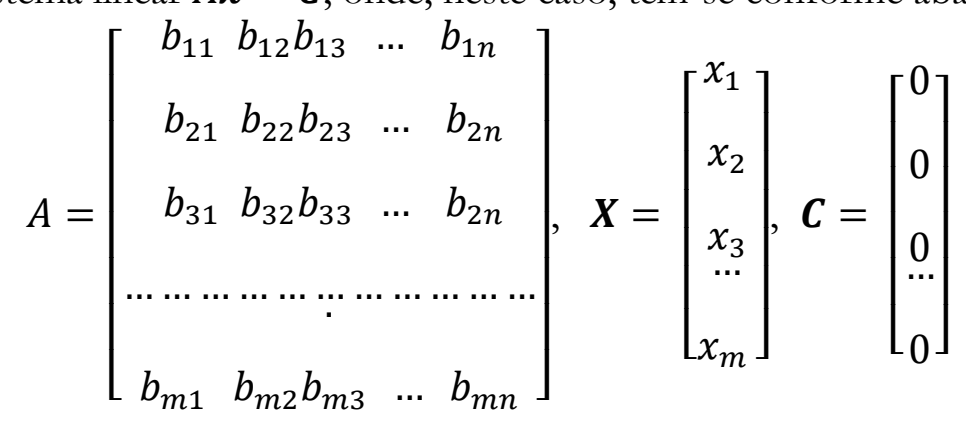


Nesta conformidade, uma solução de um sistema linear homogéneo pode ser entendida como um vector $\boldsymbol{y}=\left[\begin{array}{c}y_{1} \\ y_{2} \\ y_{3} \\ \cdots \\ y_{n}\end{array}\right]$, tal que as equações do sistema linear homogéneo sejam satisfeitas, quando se substitui $x_{1}=y_{1} ; x_{2}=y_{2} ; x_{3}=y_{3}, \ldots, x_{n}=y_{n}$.

Atendendo ao facto de equações e sistemas homogéneos desempenharem um papel essencial em toda a Álgebra Homométrica, urge apresentar aqui alguns resultados já conhecidos da Álgebra Linear sobre sistemas homogéneos, tal como segue nas proposições abaixo.

PROPOSIÇÃO 1. Seja $n$ o número de incógnitas e $m$ o número de equações em um sistema homogéneo; $A=\left(a_{i j}\right)_{m \times n}$, a matriz dos coeficientes, tal que $m<n$. Então, o sistema homogéneo $A \boldsymbol{x}=\mathbf{0}$ tem solução diferente da solução trivial.

DEMONSTRAÇÃO. Já que o sistema homogéneo considerado tem menos equações do que incógnitas $(m<n)$ e considerando o método de Gauss-Jordan, o número de linhas não nulas $r$ da forma escalonada da matriz aumentada do sistema, obtida por este método de Gauss-Jordan, é tal que $r<n$. Nestas condições, tem-se $r$ pivôs e $n-r$ incógnitas livres, passíveis de assumir qualquer valor real. Assim, o sistema admite solução não trivial, ou seja, infinitas soluções.

Esta proposição 1 garante a existência de soluções não nulas em sistemas de equações lineares homogéneos que possuem o número de incógnitas maior que o número de equações. Entretanto, não afirma nada sobre casos para os quais o número de incógnitas é igual ao número de equações, o que é garantido na proposição 2 abaixo.

PROPOSIÇÃO 2. Seja $A \boldsymbol{x}=0$ um sistema de equações lineares homogéneas qualquer, para o qual $A=\left(a_{i j}\right)_{n \times n}$ é uma matriz quadrada. Então, o sistema linear homogéneo $A \boldsymbol{x}=0$ tem somente a solução trivial.

DEMONSTRAÇÃO. Considere-se uma matriz quadrada $A=\left(a_{i j}\right)_{n \times n}$ com inversa $A^{-1}$ e que $\boldsymbol{x}_{\mathbf{0}}$ seja uma solução qualquer do sistema homogéneo $\boldsymbol{A} \boldsymbol{x}=0$. Então, multiplicando ambos os membros desta equação homogénea pela matriz inversa $A^{-1}$, tem-se:

$$
A^{-1}\left(A \boldsymbol{x}_{\mathbf{0}}\right)=\mathbf{0} A^{-1} \text {. }
$$

Desta igualdade segue a expressão abaixo, aplicando operações matriciais básicas:

$$
A^{-1}\left(A \boldsymbol{x}_{\mathbf{0}}\right)=\mathbf{0} A^{-1} \Rightarrow\left(A^{-1} A\right) \boldsymbol{x}_{0}=\mathbf{0} \Rightarrow I \boldsymbol{x}_{0}=\mathbf{0} .
$$

Desta expressão, $I$ é a matriz identidade. Daí, conclui-se, tal como se queria demonstrar, que a única solução deste sistema considerado é a solução trivial, ou seja:

$$
\boldsymbol{x}_{0}=\mathbf{0} \text {. }
$$

Por esta proposição 2 , todo o sistema de equações lineares homogéneo que apresenta o mesmo número de equações e incógnitas, ou seja, $n=m$, sendo $n$ o número de incógnitas e $m$ o número de equações, tem como única solução a solução trivial.

PROPOSIÇÃO 3. Sejam $\boldsymbol{x}_{\mathbf{0}}, \boldsymbol{x}_{\mathbf{1}}, \boldsymbol{x}_{\mathbf{2}}, \ldots, \boldsymbol{x}_{\boldsymbol{n}}$ soluções do sistema linear homogéneo $\boldsymbol{A} \boldsymbol{x}=\mathbf{0}$. Então, as seguintes afirmações são verdadeiras:

i) A soma destas soluções $\boldsymbol{x}_{\mathbf{0}}+\boldsymbol{x}_{\mathbf{1}}+\boldsymbol{x}_{\mathbf{2}}+\cdots+\boldsymbol{x}_{\boldsymbol{n}}$ também é solução deste sistema linear homogéneo $A \boldsymbol{x}=\mathbf{0}$;

ii) Para todo escalar $\alpha \in \mathbb{K}, \alpha \boldsymbol{x}_{i}, i \in \mathbb{N}$, é também solução deste sistema de equações lineares homogéneo $\boldsymbol{A x}=\mathbf{0}$. 
DEMONSTRAÇÃO. Demonstrando cada uma destas afirmações, segue:

i) Sejam $\boldsymbol{x}_{\mathbf{0}}, \boldsymbol{x}_{1}, \boldsymbol{x}_{2}, \ldots, \boldsymbol{x}_{n}$ soluções do sistema linear homogéneo $\boldsymbol{A} \boldsymbol{x}=\mathbf{0}$. Então temse:

$$
A x_{0}=A x_{1}=A x_{2}=\cdots=A x_{n}=0 .
$$

De facto, seja, por hipótese, $\boldsymbol{x}=\boldsymbol{x}_{\mathbf{0}}+\boldsymbol{x}_{\mathbf{1}}+\boldsymbol{x}_{\mathbf{2}}+\cdots+\boldsymbol{x}_{\boldsymbol{n}}$ uma solução deste sistema. Então, multiplicando ambos os membros desta igualdade pela matriz $A$, tem-se:

$$
A \boldsymbol{x}=A \boldsymbol{x}_{\mathbf{0}}+A \boldsymbol{x}_{1}+A \boldsymbol{x}_{2}+\cdots+A \boldsymbol{x}_{n} .
$$

Desta última expressão, segue, tal como se queria demonstrar que, nestas condições, $\boldsymbol{x}=$ $\boldsymbol{x}_{\mathbf{0}}+\boldsymbol{x}_{\mathbf{1}}+\boldsymbol{x}_{\mathbf{2}}+\cdots+\boldsymbol{x}_{\boldsymbol{n}}$ é solução do sistema linear homogéneo $\boldsymbol{A x}=\mathbf{0}$, ou seja:

$$
A \boldsymbol{x}=\mathbf{0}+\mathbf{0}+\mathbf{0}+\cdots+\mathbf{0} \Rightarrow A \boldsymbol{x}=\mathbf{0} \text {. }
$$

ii) Seja $\boldsymbol{x}_{\boldsymbol{i}}$ uma solução do sistema linear homogéneo $A \boldsymbol{x}=\mathbf{0}$. Então $\alpha \boldsymbol{x}_{\boldsymbol{i}}$ também é solução deste sistema, pois, tal como se queria demonstrar:

$$
A(\alpha \boldsymbol{x})=\alpha(A \boldsymbol{x})=\alpha \mathbf{0}=\mathbf{0} \text {. }
$$

Desta proposição 3 se pode verificar importante comportamento de sistemas de equações lineares homogéneos que não é satisfeito por sistemas lineares em geral de tipo $A \boldsymbol{x}=\boldsymbol{c}$, e que desempenha um papel fundamental na descrição de sub-espaços de espaços vectoriais reais $\mathrm{n}$-dimensionais. De facto, por esta proposição 3 , o conjunto solução de um sistema linear homogéneo é um sub-espaço do $\mathbb{R}^{n}$, ou seja, é um subconjunto deste espaço vectorial real $n$-dimensional que cumpre com todos os axiomas que definem um espaço vectorial, o que não acontece com o conjunto solução de um sistema linear não-homogéneo, pois não contém o vector nulo e qualquer das suas soluções é única e não admite, geralmente, sub-soluções.

\section{Definição de Equação Homogénea Geral e Equação Homogénea Linear Identitária}

$\mathrm{Na}$ literatura algébrica, uma equação homogénea linear apresenta a forma observada no item 2 que aborda sobre equação linear e sistemas lineares homogéneos (Farias et al, 2018). Entretanto, o conceito de homogeneidade é bem mais amplo e enquadra funções homogéneas, quaisquer equações lineares, equações diferenciais, havendo assim necessidade de se estabelecer uma definição de equação homogénea em geral. A rigor, a homogeneidade extrapassa qualquer ciência particular, ou seja, é uma noção transversal que exprime a ideia do que há de comum em seres, objectos, processos, métodos, tecnologias, enfim. Homogeneidade é, para além de um conceito científico, um termo filosófico, tecnológico, antropológico. A definição 1 abaixo apresenta a forma de uma equação homogénea geral.

DEFINIÇÃO 1. Seja $\mathbb{V}^{n}$ um espaço vectorial n-dimensional sobre um corpo $\mathbb{K}, F$ o conjunto de todas as funções de $\mathbb{V}^{n}$. Diz-se que $H\left(f_{i}\right)=0$ é uma equação homogénea geral definida no espaço vectorial $\mathbb{V}^{n}$ se se tem:

$$
H\left(f_{i}\right)=\sum_{i=1}^{k}\left(b_{i} f_{i}\right)=0 .
$$

Os $b_{i}$ são elementos do corpo $\mathbb{K}$ e $f_{i}$ são funções de natureza diversa pertencentes ao conjunto $F$ definido no espaço vectorial $\mathbb{V}^{n}$, para $k \in \mathbb{N}$.

Resulta desta definição 1 que uma equação homogénea geral apresenta várias configurações dependentes da tipologia de funções que a compõem, pela qual uma equação homogénea pode ser linear ou não linear. Atendendo à forma como estas funções se relacionam na equação, esta pode ser simples ou composta. Ademais, importa realçar que uma classificação geral da equação homogénea linear não cabe no escopo desta obra, estando reservada para abordagens mais aprofundadas sobre a Álgebra Homométrica.

A expressão equação homogénea linear identitária é quase toda familiar, a menos do termo identitária. Entretanto, parece fácil perceber que este termo deriva da palavra identidade e, como tal, deve estar associado às funções componentes da equação homogénea. Assim, trata-se da presença de funções 
identidades como componentes da equação homogénea, ou seja, as funções componentes consideradas são funções identidades.

Por esta explanação, uma equação homogénea linear identitária apresenta uma tipologia simples, uma vez que todas as suas funções componentes devem ser funções identidades. A definição 2 abaixo apresenta a característica fundamental destes tipos de equações, a de terem funções lineares identidades como componentes.

DEFINIÇÃO 2. Seja $\mathbb{V}^{n}$ um espaço vectorial n-dimensional sobre um corpo $\mathbb{K}, \quad L$ o conjunto de todas as funções lineares de $\mathbb{V}^{n}$. Uma equação homogénea linear identitária é uma equação homogénea linear para a qual as funções lineares componentes são funções lineares identidades, ou seja:

$$
H\left(x_{i}\right)=\sum_{i=1}^{n}\left[b_{i}\left(x_{i}\right)\right]=0 .
$$

Esta equação tem, claramente, o formato de uma equação linear homogénea definida no âmbito da Álgebra Linear, apresentada anteriormente, ou seja, a equação homogénea linear identitária pode ser escrita na forma seguinte:

$$
\sum_{i=1}^{n}\left[b_{i}\left(x_{i}\right)\right]=b_{1} x_{1}+b_{2} x_{2}+b_{3} x_{3}+\cdots+b_{n} x_{n}=0 .
$$

Neste caso, são funções componentes da equação homogénea linear identitária as funções lineares tais que:

$$
f_{1}\left(x_{1}\right)=x_{1} ; f_{2}\left(x_{2}\right)=x_{2} ; f_{3}\left(x_{3}\right)=x_{3} ; f_{4}\left(x_{4}\right)=x_{4} ; \ldots ; f_{n}\left(x_{n}\right)=x_{n} .
$$

Um conjunto de equações homogéneas lineares identitárias, também chamado, simplesmente, conjunto de equações lineares homogéneas, forma um sistema homogéneo de equações lineares, ao qual se aplicam todas as proposições e teoremas conhecidos no contexto da Álgebra Linear e da Geometria Analítica.

De facto, se $\mathbb{K}$ é um corpo de escalares e $A$ uma matriz dos coeficientes $b_{i} \in \mathbb{K}$, e $\boldsymbol{x}$ o vectorsolução, então $A \boldsymbol{x}=\mathbf{0}$ é um sistema linear homogéneo identitário na forma matricial, compacta, de cuja forma analítica, não compacta, foi apresentada anteriormente, no item que aborda sobre equações lineares e sistemas homogéneos.

\section{Equação Homogénea Linear Vectorial}

As palavras que compõem a expressão equação homogénea linear vectorial são todas familiares ao contexto da disciplina de Álgebra Linear. Contudo, a expressão como um todo designa aqui um conceito não tão familiar, pelo menos numa primeira aproximação, revelando a sua identidade após alguns resultados que serão apresentados no decorrer da abordagem deste item. A definição 3 abaixo exprime uma versão formal de uma equação homogénea linear vectorial.

DEFINIÇÃo 3. Seja $\mathbb{V}^{n}$ um espaço vectorial n-dimensional sobre o qual está fixado um ponto $P_{0}$ associado a um vector $\boldsymbol{v}_{0}=\left(a_{1} ; a_{2} ; a_{3} ; \ldots ; a_{n}\right)$, considerados constantes, podendo, a partir do ponto $P_{0}$, ser obtido um outro ponto, o ponto $P_{1}$ e, em consequência, do vector $\boldsymbol{v}_{0}$ deriva, por variação de pelo menos um dos seus componentes, o vector $\boldsymbol{v}_{1}=\left(b_{1} ; b_{2} ; b_{3} ; \ldots ; b_{n}\right)$. Seja $\boldsymbol{x}=$ $\left(x_{1} ; x_{2} ; x_{3} ; \ldots ; x_{n}\right)$ um vector deste espaço vectorial. Nestas condições, chama-se equação homogénea linear vectorial a equação de tipo

$$
\sum_{i=1}^{n}\left[x_{i}\left(b_{i}-a_{i}\right)\right]=0 \text {, }
$$


para a qual $f_{i}\left(x_{i}\right)=x_{i}\left(b_{i}-a_{i}\right)$ são as funções componentes e $a_{i}, b_{i}$, as componentes dos vectores $\boldsymbol{v}_{0}$ e $\boldsymbol{v}_{1}$, denominados vector inicial e vector pós-inicial, respectivamente, e $x_{i}$, os coeficientes incógnitas da equação.

A ideia de uma equação homogénea linear vectorial reside no facto de, dados dois vectores $\boldsymbol{v}_{0}$ e $\boldsymbol{v}_{1}$, com $\boldsymbol{v}_{0}$ fixo, é sempre possível determinar um terceiro vector $\boldsymbol{x}$ do mesmo espaço vectorial, a partir destes dois vectores exclusivamente, sendo este vector $\boldsymbol{x}$ o único vector linearmente independente associado aos dois vectores dados $\boldsymbol{v}_{0}$ e $\boldsymbol{v}_{1}$.

TEOREMA 1. Considere-se uma equação homogénea linear vectorial como a apresentada na definição 3. Então, toda equação linear, dada por uma igualdade de tipo

$$
b_{1} x_{1}+b_{2} x_{2}+b_{3} x_{3}+\cdots+b_{n} x_{n}=c,
$$

é uma equação homogénea linear vectorial, para a qual $c=a_{1} x_{1}+a_{2} x_{2}+a_{3} x_{3}+\cdots+a_{n} x_{n}$; $x_{i}$ são os coeficientes e $a_{i}, b_{i}$, componentes dos vectores inicial e pós-inicial, respectivamente, associados a um espaço vectorial $\mathbb{V}^{n}$.

DEMONSTRAÇÃO. Sabe-se que uma equação homogénea linear vectorial é da forma:

$$
x_{1}\left(b_{1}-a_{1}\right)+x_{2}\left(b_{2}-a_{2}\right)+x_{3}\left(b_{3}-a_{3}\right)+\cdots+x_{n}\left(b_{n}-a_{n}\right)=0 \text {. }
$$

Desenvolvendo o membro esquerdo desta igualdade, segue:

Ou seja:

$$
b_{1} x_{1}-a_{1} x_{1}+b_{2} x_{2}-a_{2} x_{2}+b_{3} x_{3}-a_{3} x_{3}+\cdots+b_{n} x_{n}-a_{n} x_{n}=0 \text {. }
$$

$$
b_{1} x_{1}+b_{2} x_{2}+b_{3} x_{3}+\cdots+b_{n} x_{n}=a_{1} x_{1}+a_{2} x_{2}+a_{3} x_{3}+a_{n} x_{n}
$$

A partir desta última igualdade, o membro direito - constituído por produtos de componentes do vector inicial fixo, constante, e componentes do vector dos coeficientes $\boldsymbol{x}=\left(x_{1} ; x_{2} ; x_{3} ; \ldots ; x_{n}\right)$ pode ser representado por um único parâmetro homogéneo $c$, de tal modo que se tenha a seguinte equação linear, tal como se queria demonstrar:

$\operatorname{com} c=c_{1}+c_{2}+c_{3}+\cdots+c_{n}$, e:

$$
a_{1} x_{1}+a_{2} x_{2}+a_{3} x_{3}+\cdots+a_{n} x_{n}=c
$$

$$
c_{1}=a_{1} x_{1} ; c_{2}=a_{2} x_{2} ; c_{3}=a_{3} x_{3} ; \ldots ; c_{n}=a_{n} x_{n} .
$$

O conjunto de duas ou mais equações homogéneas lineares vectoriais constitui um sistema de equações lineares homogéneo. O contrário também é verdadeiro, pelo teorema 1, pois a partir de equações lineares se pode formar equações homogéneas vectoriais, sendo esta uma técnica importante para o método de contracção dimensional sistemática abordado neste trabalho, em especial, nos itens abaixo. Nesta visão, as condições de dependência e independência de vectores aqui consideradas válidas são aquelas já conhecidas e definidas no contexto da Álgebra Linear. Ademais, tais vectores podem constituir sistemas lineares homogéneos com solução única para casos em que $m=n$, tendo tais sistemas infinitas soluções para casos em que se tem $m<n$, tal como se observa a partir da proposição 1 anteriormente considerada.

\section{Resolução de Equações Homogéneas Lineares pelo Método de Contracção Dimensional Sistemática}

O método de contracção dimensional sistemática de equações lineares aqui em análise fundamenta-se em resultados teóricos já apresentados neste trabalho nas proposições 2 e 3 , bem como no teorema 1 anteriormente considerado. A definição 4 abaixo apresenta o conceito formal deste método de contracção dimensional sistémica.

DEFINIÇÃO 4. Seja $\mathbb{V}^{n}$ um espaço vectorial n-dimensional sobre o qual está fixado um ponto $P_{0}$, a partir do qual se pode obter um outro ponto $P_{1}$, e $b_{1} x_{1}+b_{2} x_{2}+b_{3} x_{3}+\cdots+b_{n} x_{n}=c$, 
uma equação linear definida neste espaço vectorial. Então, o método $M_{i}$ diz-se método de contracção dimensional sistemática de equações lineares se, dados os vectores $\boldsymbol{v}_{0}=$ $\left(a_{1} ; a_{2} ; a_{3} ; \ldots ; a_{n}\right)$ e $\boldsymbol{v}_{1}=\left(b_{1} ; b_{2} ; b_{3} ; \ldots ; b_{n}\right), M_{i}$ permite transformar esta equação linear em um sistema homogéneo de equações lineares, pelo qual se pode calcular o vector de coeficientes $\boldsymbol{X}=\left(x_{1} ; x_{2} ; x_{3} ; \ldots ; x_{n}\right)$ associado a estes vectores dados.

No contexto desta definição 4 , os $a_{i}$ e $b_{i}$ são componentes, respectivamente, dos vectores $\boldsymbol{v}_{0}$ e $\boldsymbol{v}_{i}$, os quais são dados, restando os $x_{i}$ como incógnitas da equação. Ou seja, fixado um ponto $P_{0}$ num espaço vectorial de dimensão finita $\mathbb{V}^{n}$, caso se conheça um outro ponto $P_{1}$ deste espaço, que evolui a partir do ponto fixado $P_{0}$, então os coeficientes $x_{i}$ de uma equação homogénea linear vectorial que envolve os vectores $\boldsymbol{v}_{0}$ e $\boldsymbol{v}_{1}$ podem ser determinados pelo método de contracção sistemática de equação homogénea ou sistema homogéneo em consideração (Cunha, 2009).

TEOREMA 2. Seja

$$
b_{1} x_{1}+b_{2} x_{2}+b_{3} x_{3}+\cdots+b_{n} x_{n}=c
$$

uma equação homogénea linear vectorial definida num espaço vectorial $\mathbb{V}^{n}$ e associada aos vectores $\boldsymbol{v}_{0}=\left(a_{1} ; a_{2} ; a_{3} ; \ldots ; a_{n}\right)$ e $\boldsymbol{v}_{1}=\left(b_{1} ; b_{2} ; b_{3} ; \ldots ; b_{n}\right)$, dados e conhecidos deste espaço. Então, as incógnitas $x_{i}$ são determinadas a partir do sistema abaixo:

$$
\left\{\begin{array}{l}
a_{1} x_{1}+a_{2} x_{2}+a_{3} x_{3}+\cdots+a_{n} x_{n}=0 \\
b_{1} x_{1}+b_{2} x_{2}+b_{3} x_{3}+\cdots+b_{n} x_{n}=0
\end{array} .\right.
$$

DEMONSTRAÇÃO. Considere-se a equação linear apresentada neste teorema:

$$
b_{1} x_{1}+b_{2} x_{2}+b_{3} x_{3}+\cdots+b_{n} x_{n}=c \quad(2) \text {. }
$$

A partir desta equação linear, identifica-se o parâmetro homogéneo $c$, tal que

e que:

$$
c_{1}=a_{1} x_{1} ; c_{2}=a_{2} x_{2} ; c_{3}=a_{3} x_{3} ; \ldots ; c_{n}=a_{n} x_{n},
$$

$$
c=c_{1}+c_{2}+c_{3}+\cdots+c_{n} \Rightarrow c=a_{1} x_{1}+a_{2} x_{2}+a_{3} x_{3}+\cdots+a_{n} x_{n} .
$$

Substituindo esta última igualdade na equação (2), segue:

$$
b_{1} x_{1}+b_{2} x_{2}+b_{3} x_{3}+\cdots+b_{n} x_{n}=a_{1} x_{1}+a_{2} x_{2}+a_{3} x_{3}+\cdots+a_{n} x_{n} .
$$

Organizando esta igualdade, tem-se:

$$
b_{1} x_{1}-a_{1} x_{1}+b_{2} x_{2}-a_{2} x_{2}+b_{3} x_{3}-a_{3} x_{3}+\cdots+b_{n} x_{n}-a_{n} x_{n}=0 \text {. }
$$

Um olhar sobre esta expressão permite interpretá-la como a soma de duas equações lineares

$$
\begin{aligned}
& b_{1} x_{1}+b_{2} x_{2}+b_{3} x_{3}+\cdots+b_{n} x_{n}=0 \mathrm{e} \\
& -a_{1} x_{1}-a_{2} x_{2}-a_{3} x_{3}-\cdots-a_{n} x_{n}=0
\end{aligned}
$$

de incógnitas $x_{1}, x_{2}, x_{3}, \ldots, x_{n}$. Considere-se que tal soma seja operada por conta do uso do método de adição associado à resolução de sistemas de equações lineares na disciplina de Álgebra Linear (Anton e Rorres, 2012). Por conseguinte, resulta fácil retornar ao sistema de equações lineares de que resultou a referida equação, pelo que, tal como se queria demonstrar, segue:

$$
\left\{\begin{array}{l}
a_{1} x_{1}+a_{2} x_{2}+a_{3} x_{3}+\cdots+a_{n} x_{n}=0 \\
b_{1} x_{1}+b_{2} x_{2}+b_{3} x_{3}+\cdots+b_{n} x_{n}=0
\end{array}\right. \text {. }
$$

EXEMPLO 1. Sejam $\boldsymbol{v}_{0}=(3 ; 4 ; 7)$ e $\boldsymbol{v}_{1}=(9 ; 5 ; 9)$ vectores de $\mathbb{V}^{3}$. Encontre:

a) A equação homogénea linear;

b) O sistema de equações homogéneas lineares associado.

\section{Resolução}

a) Considerando a equação homogénea linear, segue:

$$
9 x_{1}+5 x_{2}+9 x_{3}=c \text { e } c=3 x_{1}+4 x_{2}+7 x_{3} \text {. }
$$


Então, a equação homogénea linear correspondente será:

$$
6 x_{1}+x_{2}+2 x_{3}=0 \text {. }
$$

b) Considerando esta equação homogénea linear, tem-se o seguinte sistema:

$$
\left\{\begin{array}{l}
9 x_{1}+5 x_{2}+9 x_{3}=0 \\
3 x_{1}+4 x_{2}+7 x_{3}=0
\end{array}\right. \text {. }
$$

Usando qualquer método clássico directo percebe-se que, para este sistema linear, $x_{1}=-1, x_{2}=$ $-36, x_{3}=21$ é uma solução. E esta solução vale para a equação homogénea apresentada e para o correspondente sistema homogéneo em estudo.

É claro que este conjunto-solução depende dos valores dos componentes dos vectores dados, ou seja, fixado o vector inicial, o vector $\boldsymbol{x}=\left(x_{1} ; x_{2} ; x_{3} ; \ldots ; x_{n}\right)$ dependerá da evolução do sistema que integra os dois pontos, vectores considerados. Neste sentido, a equação linear dada relaciona pura e simplesmente os dois vectores dados, não dependendo de qualquer outro factor, um indício importante do carácter dinâmico de que se reveste a Álgebra Homométrica. Um desenvolvimento mais aprofundado deste facto, como por exemplo, análise algébrica tetradimensional ou pentadimensional da dinâmica de pontos com simuladores informáticos será desenvolvida em outros trabalhos mais específicos.

No contexto da Mecânica Clássica, por exemplo, um ponto material que se desloca sobre a superfície de um corpo, dentro de um dado campo gravitacional, com condições iniciais de movimento dinâmico bem definidas, tais como força de atrito e coeficiente de atrito, massa, força de gravidade, força de reacção normal, possui um vector dinâmico inicial característico do ponto material e do meio com o qual interage, constituído pelos valores destas grandezas do estágio inicial. Este vector pode evoluir para um vector pós-inicial, na medida em que pelo menos uma destas grandezas pode variar. Dados estes dois vectores, pode-se determinar o valor do vector de coeficientes $\boldsymbol{x}=\left(x_{1} ; x_{2} ; x_{3} ; \ldots ; x_{n}\right)$, do qual dependem grandezas dinâmicas importantes como aceleração, força resultante, força de inércia, dentre outras.

\section{Resolução de Sistemas Homogéneos de duas Equações Lineares pelo Método de Contracção Dimensional Sistemática}

Este trabalho parte do princípio segundo o qual o teorema de Crámer aplicado a qualquer sistema homogéneo de duas equações lineares com três incógnitas permite encontrar soluções do referido sistema, considerando $x_{3}=\Delta$, onde $x_{3}$ é a terceira incógnita das equações deste sistema e $\Delta$, o determinante relativo às duas incógnitas iniciais, ou seja:

$$
\begin{gathered}
\left\{\begin{array} { l } 
{ a _ { 1 } x _ { 1 } + a _ { 2 } x _ { 2 } + a _ { 3 } x _ { 3 } = 0 } \\
{ b _ { 1 } x _ { 1 } + b _ { 2 } x _ { 2 } + b _ { 3 } x _ { 3 } = 0 }
\end{array} \rightarrow \left\{\begin{array}{l}
a_{1} x_{1}+a_{2} x_{2}=-a_{3} x_{3} \\
b_{1} x_{1}+b_{2} x_{2}=-b_{3} x_{3}
\end{array}\right.\right. \\
\Rightarrow \Delta=a_{1} b_{2}-a_{2} b_{1} \text { e } x_{3}=a_{1} b_{2}-a_{2} b_{1} .
\end{gathered}
$$

A presente proposta metodológica de contracção dimensional sistemática centra-se na redução do número de incógnitas até atingir apenas três incógnitas, visando cumprir o princípio acenado acima, a partir do qual se tem a solução que se observará abaixo (Miranda et al, 2015).

Nesta conformidade, o método de contracção dimensional sistemática pode ser descrito através de suas quatro principais características que o diferenciam dos demais métodos de resolução de sistemas lineares:

1.Reduz, sistematicamente, o número de incógnitas de um sistema de equações lineares homogéneas até um número de incógnitas mais favorável à sua resolução;

2.Converte sistemas lineares não-homogéneos em sistemas lineares homogéneos;

3.Permite converter uma equação linear em sistema de equações lineares homogéneo; 
4. Garante soluções de sistemas lineares homogéneos, em geral, originais e exactas, sem recurso a variáveis livres que podem assumir quaisquer valores, no contexto dos métodos de escalonamento;

5.Permite obter uma solução padrão única (solução linearmente independente) de um sistema linear homogéneo que, no campo da Álgebra Linear, possui infinitas soluções.

De facto, no quadro da Álgebra Linear, a resolução de sistemas homogéneos de equações lineares com $n$ incógnitas e $m$ equações e que contam com soluções não triviais, em conformidade com a proposição 1, contempla a existência de variáveis livres que exigem atribuição aleatória de valores do corpo de escalares para se obter as soluções respectivas, uma situação que omite o verdadeiro formato, espectro, forma ou aspecto formal das soluções do sistema em resolução.

Para ultrapassar esta dificuldade e garantir o aspecto formal real das soluções de sistemas homogéneos existe o método de contracção sistemática de equações lineares e sistemas homogéneos de equações lineares que segue definido, na definição 5 abaixo, e posteriormente analisado na sua operacionalização.

DEFINIÇÃO 5. Seja $A \boldsymbol{x}=\mathbf{0}$ um sistema homogéneo de equações lineares que admite soluções não triviais. Então, o método $M_{i}$ diz-se método de contracção dimensional sistemática de sistemas lineares homogéneos se, na busca por soluções deste sistema, $M_{i}$ expande o sistema $A \boldsymbol{x}=\mathbf{0}$ com $n$ incógnitas e $m$ equações em um somatório de vários sistemas homogéneos associados $A \boldsymbol{x}_{1}+A \boldsymbol{x}_{2}+A \boldsymbol{x}_{3}+\cdots+A \boldsymbol{x}_{n-q}=\mathbf{0}$, contraindo sistematicamente o número de incógnitas da equação linear de $n$ para $n-1$, de $n-1$ para $n-2$, e assim por diante.

O método de contracção dimensional sistemática aplica-se a sistemas de 2, 3, 4 ou mais equações lineares, cujas soluções são não triviais, pois para casos de soluções triviais referenciadas na proposição 1, a solução é conhecida a priori, não havendo necessidade de qualquer cálculo. Entretanto, no contexto deste trabalho, em particular, este método será aplicado apenas a sistemas homogéneos de duas equações lineares, ficando reservado para análises futuras a aplicação deste método para sistemas com mais de duas equações. Em casos de existir apenas uma equação linear, utiliza-se primeiro o método de contracção dimensional sistemática de equações lineares para depois, obtido um sistema homogéneo, aplicar o método de contracção sistemática de sistemas de equações lineares.

O método de contracção dimensional sistemática aplica-se a sistemas de duas equações lineares com mais de 3 incógnitas, uma vez que, tal como já foi demonstrado na proposição 1, os sistemas com duas incógnitas e duas equações têm apenas a solução trivial, e os sistemas com 3 incógnitas que tenham soluções não triviais podem ser resolvidos com os métodos clássicos conhecidos na Álgebra Linear, conservando-se toda a estrutura das suas soluções originais.

Por conta desta proposição 1, os sistemas com 4 ou mais incógnitas também podem apresentar apenas a solução trivial, desde que tenham o mesmo número de incógnitas e equações, devendo este método em referência ser aplicado, de modo eficiente, a sistemas homogéneos com soluções não triviais.

Assim, os sistemas homogéneos de duas equações lineares com 4 ou mais incógnitas que apresentam soluções não triviais podem ser resolvidos por este método de contracção sistemática, aplicando-se os seguintes passos:

i) À luz da proposição 3, cada $\boldsymbol{x}_{i}$ de cada equação linear dada deve ser decomposto de tal modo que a solução geral $\boldsymbol{x}$ seja composto pela soma das soluções particulares $\boldsymbol{x}_{i j}$ de cada sub-espaço de dimensão $n-1$ que compõe o espaço vectorial $\mathbb{V}^{n}$ considerado;

ii) O número de soluções particulares referentes aos sub-espaços de dimensão $n-1$ de $\mathbb{V}^{n}$ são em número de $n-1$, sendo $n$ a dimensão do espaço vectorial em consideração; 
iii) Em seguida, forma-se, para cada uma das duas equações dadas, um sistema homogéneo com $n$ equações lineares e $n-1$ incógnitas, obtendo-se, assim dois sistemas homogéneos de equações lineares em que se repetem em cada coluna as componentes dos vectores associados; iv) O passo seguinte será a formação de sistemas homogéneos parciais de duas equações lineares para cada sistema, seleccionadas por formas a se obter uma equação do primeiro sistema homogéneo formado a partir da primeira equação linear do sistema original dado, e uma equação do segundo sistema homogéneo formado a partir da segunda equação linear do sistema original dado;

v) Cada sistema homogéneo parcial assim obtido terá $n-1$ incógnitas e as suas equações serão seleccionadas de tal modo que se tenha, em cada sistema homogéneo parcial, os mesmos coeficientes originais dados;

vi) Se os sistemas homogéneos parciais obtidos têm mais de 3 incógnitas, de tal modo que não possam ser resolvidos com os métodos clássicos conhecidos, então o processo prossegue, repetindo os cinco primeiros passos;

vii) Para cada repetição dos cinco primeiros passos, os coeficientes das equações lineares de cada sistema expandido resultante deverão ser decompostos, em conformidade com a proposição 3.

Mais abaixo segue um exemplo de aplicação deste método de contracção dimensional sistemática para o cálculo de um sistema linear homogéneo de duas equações e 4 incógnitas. Em trabalhos futuros serão desenvolvidos outros mais exemplos aplicados a campos como Física, Química, Biologia, Ciências da Computação e Cosmologia, um escopo que o carácter básico deste trabalho não permite. Destarte, dado um sistema de equações lineares homogéneo como o apresentado acima, com $n>m$, onde $n$ é o número de incógnitas e $m$ o número de equações, a sua resolução, através do método de contracção dimensional sistemática, segue os passos acima, os quais podem ser resumidos como segue:

1. Decompor cada incógnita do sistema de equações lineares dado como soma de $n-1$ novas variáveis;

2. Substituir as novas variáveis no sistema de equações lineares dado para cada equação deste sistema;

3. Formar novos sistemas de equações lineares com $m$ equações, que contenham variáveis da mesma natureza, cujo número de incógnitas esteja reduzido para $n-1$, chamados sistemas parciais;

4. Repetir este processo até que os sistemas parciais tenham $n=3$ incógnitas;

5. Resolver os sistemas parciais com 3 novas incógnitas e substituir os seus valores na soma que compõe as incógnitas originais para determinar os valores destas incógnitas originais.

Para $n=4$, o método de contracção dimensional sistemática deve ser aplicado de tal modo que se possa reduzir o número de incógnitas até $n-1$, e neste caso, até $n=3$, o que significa que cada incógnita de (3) deverá ser decomposta em 3 sub-incógnitas, ou seja:

$$
\left\{\begin{array}{l}
x_{1}=x_{11}+x_{12}+x_{13} \\
x_{2}=x_{21}+x_{22}+x_{23} \\
x_{3}=x_{31}+x_{32}+x_{33} \\
x_{4}=x_{41}+x_{42}+x_{43}
\end{array}\right.
$$

Aplicando esta decomposição da incógnita, primeiro para a primeira equação e depois para a segunda equação do sistema (3), tem-se:

$$
\text { I) }\left\{\begin{array}{c}
3 x_{11}+x_{21}+2 x_{31}+0=0 \\
3 x_{12}+x_{22}+0-4 x_{41}=0 \\
3 x_{13}+0+2 x_{32}-4 x_{42}=0 \\
0+x_{23}+2 x_{33}-4 x_{43}=0
\end{array}\right.
$$


De facto, se se aplicar o método de substituição para este sistema acima, ter-se-á a equação I com todas as suas novas incógnitas.

Para a segunda equação do sistema (1), segue:

$$
\text { II) }\left\{\begin{array}{c}
2 x_{11}+2 x_{21}-3 x_{31}+0=0 \\
2 x_{12}+2 x_{22}-0+x_{41}=0 \\
2 x_{13}+0-3 x_{32}+x_{42}=0 \\
0+2 x_{23}-3 x_{33}+x_{43}=0
\end{array}\right.
$$

A partir destes sistemas expandidos I) e II), são formados os quatro sistemas parciais seguintes que juntam uma equação do sistema I) com uma equação do sistema II), contendo as mesmas incógnitas, ou seja:

$$
\begin{gathered}
\left\{\begin{array}{l}
3 x_{11}+x_{21}+2 x_{31}=0 \\
2 x_{11}+2 x_{21}-3 x_{31}=0
\end{array} ; b\right)\left\{\begin{array}{l}
3 x_{12}+x_{22}-4 x_{41}=0 \\
2 x_{12}+2 x_{22}+x_{41}=0
\end{array} ;\right. \\
\text { c) }\left\{\begin{array} { l } 
{ 3 x _ { 1 3 } + 2 x _ { 3 2 } - 4 x _ { 4 2 } = 0 } \\
{ 2 x _ { 1 3 } - 3 x _ { 3 2 } + x _ { 4 2 } = 0 }
\end{array} ; \text { d } \left\{\begin{array}{l}
x_{23}+2 x_{33}-4 x_{43}=0 \\
2 x_{23}-3 x_{33}+x_{43}=0
\end{array}\right.\right.
\end{gathered}
$$

Resolvendo estes sistemas parciais a), b), c) e d) usando qualquer método conhecido na Álgebra Linear, tem-se as seguintes soluções:

$$
\left\{\begin{array}{l}
x_{1}=-7+9-10 \Rightarrow x_{1}=-8 \\
x_{2}=13-11-10 \Rightarrow x_{2}=-8 \\
x_{3}=4-11-9 \Rightarrow x_{3}=-16 \\
x_{4}=4-13-7 \Rightarrow x_{4}=-16
\end{array} .\right.
$$

De facto, é fácil verificar que os valores $x_{1}=-8 ; x_{2}=-8 ; x_{3}=-16 ; x_{4}=-16$ satisfazem o sistema homogéneo de duas equações lineares dado.

Contudo, este método de contracção dimensional sistemática de sistemas homogéneos pode ser bastante complexo para dimensões muito grandes de espaços vectoriais e, parece tentadora a constatação segundo a qual resulta mais simplificado resolver sistemas homogéneos lineares utilizando os métodos clássicos já conhecidos. Entretanto, a vantagem fundamental deste método de contracção dimensional sistemática está no facto de revelar a estrutura completa e formal das soluções gerais obtidas destes sistemas homogéneos lineares, uma condição sine qua non para a definição e o estudo das propriedades do produto vectorial homogéneo discutido nos próximos trabalhos.

\section{Solução-Padrão de uma Equação Homogénea Linear}

Pela proposição 3, se $\boldsymbol{x}$ é uma solução do sistema homogéneo de equações lineares $\boldsymbol{A} \boldsymbol{x}=\mathbf{0}$, então o seu produto por uma constante real também é solução deste sistema e é sempre possível encontrar subsistemas

$$
A \boldsymbol{x}_{0}=\mathbf{0}, A \boldsymbol{x}_{1}=\mathbf{0}, A \boldsymbol{x}_{2}=\mathbf{0}, \ldots, A \boldsymbol{x}_{n}=\mathbf{0} \text {, }
$$

para os quais $\boldsymbol{x}_{0}+\boldsymbol{x}_{1}+\boldsymbol{x}_{2}+\cdots+\boldsymbol{x}_{n}=\boldsymbol{x}, n \in \mathbb{N}$.

Este resultado mostra a necessidade de se introduzir o conceito de solução padrão de uma equação homogénea linear, visando identificar uma solução-modelo que deve servir propósitos específicos, já que várias soluções, diferentes no seu conteúdo, podem ser obtidas para um mesmo sistema homogéneo de equações lineares. A definição 6 abaixo apresenta, formalmente, este conceito bastante importante para, por exemplo, definir e operacionalizar o produto vectorial homogéneo, posteriormente analisado.

DEFINIÇÃO 6. Considere-se uma solução $\alpha \boldsymbol{x}$ de um sistema homogéneo de equações lineares $A \boldsymbol{x}=\mathbf{0}$ que admite soluções não triviais. Então, a solução $\boldsymbol{x}$, mais simples desta solução geral 
$\alpha \boldsymbol{x}$, de um sistema homogéneo de equações lineares é chamada de solução padrão deste sistema $A \boldsymbol{x}=\mathbf{0}$, e $\alpha$ é chamado coeficiente de expansão do sistema homogéneo $A \boldsymbol{x}=\mathbf{0}$, em relação ao sistema homogéneo tridimensional para o qual $n=3$.

Por esta definição 6 , o número $\alpha$ é sempre um número natural, pois a expansão de um dado sistema homogéneo de equações lineares somente pode ser feita em um número inteiro positivo de vezes. O teorema 3 abaixo apresenta uma forma de calcular o coeficiente de expansão $\alpha$ de um sistema homogéneo de equações lineares.

TEOREMA 3. Seja $\boldsymbol{x}$ uma solução padrão de um sistema homogéneo $A \boldsymbol{x}=\mathbf{0}$ de equações lineares com $n$ incógnitas. Então, o coeficiente de expansão $\alpha$ deste sistema homogéneo de equações lineares é dado pela seguinte igualdade:

$$
\alpha=n-3 \text {. }
$$

DEMONSTRAÇÃO. Considere-se um sistema homogéneo de equações lineares expandido $\alpha$ vezes, ou seja, $A(\alpha \boldsymbol{x})=\mathbf{0}$. Então, por hipótese, seja $\alpha=n-k$. Então, basta mostrar que $k=$ 3. Para o efeito, substituindo $\alpha=n-k$ na equação homogénea considerada, segue:

$$
A[(n-k) \boldsymbol{x}]=\mathbf{0} \text {. }
$$

Ou seja:

$$
n A \boldsymbol{x}-k A \boldsymbol{x}=\mathbf{0} \Rightarrow n A \boldsymbol{x}=k A \boldsymbol{x} .
$$

Como, segundo este método de contracção dimensional, a solução de qualquer sistema homogéneo de duas equações lineares é encontrada para $n=3$, pois em sistemas lineares homogéneos com 3 incógnitas são é necessário recorrer a variáveis livres, então o número de expansões do sistema $A \boldsymbol{x}=\mathbf{0}$ para $n=3$ e $m=2$ é nulo, ou seja, para $n=3$ e $m=2$ não existe qualquer necessidade de expansão deste sistema homogéneo.

Assim, substituindo nesta hipótese acima $n=3 \Leftrightarrow \alpha=0$ (quando $n$ é igual a 3, $\alpha$ deve necessariamente ser igual a zero), ou seja, na igualdade $\alpha=n-k$, tem-se, tal como se queria demonstrar:

$$
\alpha=n-k \Rightarrow 0=3-k \Rightarrow k=3 \text {. }
$$

Por este teorema 3, um sistema homogéneo de duas equações lineares com 4 incógnitas tem um coeficiente $\alpha$ igual a 1, ou seja, $\alpha=1 \Leftrightarrow n=4$; para um sistema homogéneo de duas equações lineares com 5 incógnitas, $\alpha$ é igual a 2, ou seja, $\alpha=2 \Leftrightarrow n=5$, e assim por diante, dependendo, o coeficiente de expansão $\alpha$, da dimensão do espaço vectorial $\mathbb{V}^{n}$ considerado. Ademais, por conta da natureza deste trabalho, o enquadramento desta temática nos procedimentos didácticos e metodológicos relativos ao cálculo de equações e sistemas de m equações lineares com $n$ incógnitas serão objecto de uma discussão futura.

\section{Considerações Finais}

O método de contracção dimensional sistemática, aqui proposto, destina-se ao cálculo de equações e sistemas de equações homogéneos lineares contraindo sistematicamente o número de incógnitas para reduzir a complexidade de cálculo deste sistema, visando determinar um vector simultaneamente ortogonal a todos os vectores multiplicativos constituintes do sistema homogéneo dado, dentre outras propriedades associadas a este vector em relação a outros vectores do mesmo espaço vectorial (Pinho, 2010).

Realça-se, assim, que o referido método não está vinculado ao produto vectorial externo, por conta da sua própria definição, o qual requer que o vector-solução e os vectores multiplicativos pertençam a bases de mesma dimensão, e não de algum espaço vectorial externo, como se verifica no produto vectorial de Gibbs e Heaviside e no produto exterior de Grassmann (Silva, 2002). Ademais, este método é essencialmente aplicado para a determinação do produto vectorial 
homogéneo associado a espaços de quaisquer dimensões, contrariamente ao que acontece com o produto externo.

Por conseguinte, o método de contracção dimensional constitui, propriamente, um método de cálculo de equações lineares, sistemas de equações lineares, sistemas homogéneos de equações lineares, cujo coeficiente de expansão seja $\alpha \geq 1$, o que ocorre para um número de incógnitas $n \geq$ 4. Destarte, este método de contracção dimensional é empregue para os seguintes casos:

i) Resolução de equações lineares, em especial, as equações lineares com mais de três incógnitas;

ii) Resolução de sistemas de equações lineares, em particular, os sistemas de equações lineares com soluções não triviais;

iii) Resolução de sistemas homogéneos de equações lineares, em especial, os sistemas homogéneos com mais de três incógnitas;

iv) Estudo do produto vectorial homogéneo, em especial para espaços vectoriais de dimensões superiores a três;

v) Estudo de triproduto, tetraproduto, pentaproduto e k-produto vectorial homogéneo;

vi) Estudo de k-produtos escalares homogéneos de vectores de espaços vectoriais ndimensionais;

vii) Estudo, em geral, da Álgebra Homométrica, Geometria Homométrica, Cálculo Diferencial e Integral Homométrico, Equações Diferenciais Homométricas e outros campos cujos objectos exigem o conhecimento da estrutura real e/ou complexa do espaço n-dimensional, planos homogéneos e sua dinâmica.

Portanto, ao longo desta abordagem apresentaram-se:

1. Fundamentos do método de contracção dimensional sistemática como método geral e apropriado para o cálculo de equações e sistemas de equações lineares homogéneos, visando soluções exactas e originais;

2. Bases metodológicas para definição e operacionalização do produto vectorial homogéneo que serve de alicerce para a construção da Álgebra Homométrica;

3. A proposta metodológica de contracção dimensional sistemática que, atendendo ao seu carácter de recorrência, é útil para a resolução de sistemas lineares através de programas computacionais de forma mais eficiente, quando são necessárias resoluções exactas destes sistemas com um número elevado de incógnitas.

\section{Referências Bibliográficas}

Anton, Howard e Rorres, Chris (2012). Álgebra linear com aplicações. Tradução de Claus Ivo Doering. Bookman. Brasil.

Cabral, Marco e Goldfeld, Paulo (2012). Curso de Álgebra Linear. Universidade Federal de Rio de Janeiro. Brasil.

Farias, Diego Marcon; Konzen, Pedro Henrique de Almeida; Souza, Rafael Rigão (2018). Álgebra Linear. Universidade Federal do Rio Grande do Sul. Brasil.

Gonçalves, Ricardo Jorge Castro (2018). Álgebra Linear. Edições Sílabo. Portugal.

Miranda, Daniel; Grisi, Rafael; Lodovici, Simué (2015). Geometria Analítica e Vectorial. Universidade Federal do ABC. Brasil.

Pinho, Domingos Pires Valente Sevivas (2010). Matrizes e aplicações no ensino secundário.

Dissertação de Mestrado. Mestrado em Matemática/Educação. Universidade Portucalense Infante D. Henrique. Portugal.

Santana, Cláudia Ribeiro; Yartey, Joseph Nee Anyah (2008). Álgebra Linear. Universidade Estadual de Santa Cruz e Universidade Federal de Bahia. Brasil.

Santos, Reginaldo J. (2010). Introdução à Álgebra Linear. Universidade Federal de Minas Gerais. ICEx. Brasil. 
Silva, Cibelle Celestino (2002). Da força ao tensor: evolução do conceito físico e da representação matemática do campo electromagnético. Tese de Doutoramento. Doutoramento em Ciências Físicas. Instituto de Física Gleb Wataghin da Universidade de Campinas. UNICAMP. Brasil.

Silva, Diego Adriano; Brito, Arnaldo Silva; Sousa, Valdirene Gomes de (2020). Equações Diafantinas Lineares: um estudo com estudantes do $1^{\circ}$ ano do Ensino Médio. Revista Electrónica da Matemática. Volume 6, No. 2. Brasil (Pp. 16).

Steinbruch, Alfredo; Winterle, Paulo (1995). Álgebra Linear. Pearson. Brasil. 